\title{
Child-Pugh versus MELD score in predicting survival in patients undergoing transjugular intrahepatic portosystemic shunt
}

\author{
B Angermayr, M Cejna, F Karnel, M Gschwantler, F Koenig, J Pidlich, H Mendel, \\ L Pichler, M Wichlas, A Kreil, M Schmid, A Ferlitsch, E Lipinski, H Brunner, J Lammer, \\ P Ferenci, A Gangl, M Peck-Radosavljevic
}

See end of article for authors' affiliations

Correspondence to: Dr M Peck-Radosavljevic, University of Vienna Medical School, Department of Medicine 4, Division of

Gastroenterology and

Hepatology, Waehringer Guertel 18-20, A-1090

Vienna, Austria;

markus.peck@akh-wien.ac.at

Accepted for publication 7 December 2002

Background: In patients undergoing transjugular intrahepatic portosystemic shunt (TIPS), prognostic scores may identify those with a poor prognosis or even those with a clear survival benefit. The ChildPugh score (CPS) is well established but several drawbacks have led to development of the model of end stage liver disease (MELD).

Aim: The aim of the study was to compare the predictive power of CPS and MELD, to validate the original MELD formula, and to assess the predictive value of the determinants used in the two prognostic scores outside of a study setting.

Patients: A total of 501 patients underwent elective TIPS placement and 475 patients fulfilled the inclusion criteria.

Methods: Data of all patients undergoing elective TIPS in one university hospital and four community hospitals in Vienna, Austria, between 1991 and 2001, were analysed retrospectively. The main statistical tests were Cox proportional hazards regression model, the log rank test, Kaplan-Meier analysis, and concordance c statistics.

Results: Median follow up was 5.2 years and median survival was 4.6 years. During follow up, 230 patients died, 75 within three months after TIPS placement. In stepwise proportional hazards analyses, independent predictors of death were creatinine level, bilirubin level, age, and refractory ascites. MELD was better in predicting survival in a stepwise Cox model but both scores were equally predictive in c statistics for one month, three month, and one year survival. Renal function was the strongest independent predictor of survival.

Conclusions: Although MELD was the primary predictor of overall survival in multivariate analysis, c statistics showed that both scores can be used for patients undergoing TIPS with equal accuracy. For assessing prognosis in patients undergoing TIPS implantation, there seems little reason to replace the well established Child-Pugh score.

mplantation of a transjugular intrahepatic portosystemic shunt (TIPS) is a well established therapy for patients with portal hypertension complicated by refractory acute variceal haemorrhage, variceal rebleeding despite endoscopic therapy, and ascites unresponsive to diuretic therapy. ${ }^{1-6}$ Patients undergoing TIPS belong to a subgroup of patients with advanced stage liver disease and at least one episode of severe decompensation. These patients have a poor prognosis. ${ }^{7}$ Since TIPS has been introduced, several factors predictive of poor survival have been recognised: hyperbilirubinaemia, hyponatraemia, episodes of hepatic encephalopathy without any triggering events, advanced liver disease, urgent TIPS placement, and non-alcoholic cirrhosis stadium Child C. ${ }^{8-13}$

A prognostic score accurately predicting survival after TIPS placement could stratify patients into those with a clear survival benefit and others who should probably not undergo TIPS placement. Many prognostic models for patients with cirrhosis have been developed. Currently, two scores are mainly used in clinical practice for patient counselling, clinical decision making, and stratifying risk in therapeutic trials.

The Child-Turcotte-Pugh (CPS) classification is by far the most widely applied and reported system as it is easy to use as a bedside test. ${ }^{14}$ It contains five variables including serum levels of bilirubin and albumin, prothrombin time, ascites, and encephalopathy. The two clinical determinants are based on subjective assessment and can be altered by therapy. CPS divides patients into low (class A), intermediate (class B), and poor (class C) risk within 10 levels of difference between the least sick patient and the most advanced. While the development of the CPS classification was based on empiric assessment, many subsequent studies have shown that CPS is predictive in the assessment of prognosis in patients with liver disease. These studies demonstrated that each of the five individual clinical variables as well as the overall CPS classification had prognostic significance. ${ }^{47^{15-19}}$ The difficulties and interobserver variability for the subjective parameters in the CPS classification led to the development of the "model for end stage liver disease" (MELD) score based on laboratory values only, which should be more objective and accurate than CPS.

Originally, the MELD score was developed for patients undergoing TIPS (TIPS-MELD score (TMS)). ${ }^{20}$ It was then modified slightly to predict survival in patients with liver cirrhosis in general (MELD). ${ }^{21}$ MELD is a continuous function of bilirubin, international normalised ratio (INR), and creatinine to predict short term (three months) survival rates and was derived by Cox proportional hazards regression analysis and validated in an independent patient sample. MELD appears to

Abbreviations: TIPS, transjugular intrahepatic portosystemic shunt; CPS, Child-Pugh score; MELD, model for end stage liver disease; TMS, TIPS-MELD score; INR, international normalised ratio; ROC, receiver operating characteristic curve. 
be reliable as a prognostic model for patients with end stage liver disease due to objective and readily available laboratory variables.

Both scores were shown to predict survival in patients undergoing TIPS

The aim of the study was to compare the predictive power of CPS, MELD, and TMS in assessment of short term and overall survival after TIPS placement, to validate the original MELD formula with our patient data, and to assess the predictive value of the determinants used in the two prognostic scores.

\section{PATIENTS AND METHODS}

Five centres offer TIPS placement in Vienna, Austria (Allgemeines Krankenhaus, Kaiser- Franz-Josef Spital, Krankenanstalt Rudolfstiftung, Krankenhaus Lainz, and Donauspital). The list of patients who underwent TIPS from May 1991 to December 2001 was retrieved from the databases of the participating hospitals. Each individual hospital record was reviewed to verify the diagnosis and indication for TIPS, and to obtain all relevant clinical and laboratory data.

Elective TIPS placement was defined as TIPS performed for the prevention of variceal rebleeding or for treatment of refractory ascites. Patients in whom TIPS was performed for the control of active variceal bleeding after failure of two sessions of endoscopic therapy within a 24 hour period were designated emergency TIPS. Refractory ascites was defined as ascites that required paracentesis for control despite a sodium restricted diet and intensive diuretic therapy, consistent with the consensus statement. ${ }^{22}$ The diagnosis of cirrhosis was confirmed by biopsy or was based on clinical, radiological, and laboratory findings. Prothrombin time, originally measured as per cent of an internal reference standard (Normotest; Nycomed), was converted to seconds and to INR using standard formulae. ${ }^{23}$ Patients with hepatic encephalopathy did not undergo TIPS.

During the study period, 523 patients underwent TIPS implantation. Twenty two patients had emergency TIPS placement and were therefore excluded from the analysis. Patients with severe infection, malignant tumours, organic renal disease, or significant cardiopulmonary comorbidity were excluded from the analysis, as well as patients without cirrhosis $^{24} 25$ and those younger than 18 years $(n=26)$. Thus a total of 475 patients were included in the analysis. Clinical and laboratory parameters were recorded within 72 hours before TIPS implantation (table 1). Complete data were not available in all patients, due to variablilty in the quality of patient documentation or lack of data within 72 hours before TIPS implantation. The number of patients available for each analysis can be seen in table 1 and in the individual statistical analyses. In all patients in whom CPS was available, the MELD score could also be computed. Missing data were evenly distributed among the participating hospitals. The TIPS procedures were performed using standard techniques. ${ }^{1326} 27$ Patients were followed from their date of TIPS until death, liver transplantation, or study closure. Survival data of all patients included into the analyses $(n=475)$ were obtained from the national death index (Statistik Austria) which keeps accurate and up to date records of all demographic data in Austria.

\section{Statistical analysis}

The starting time for all survival analyses was the date of TIPS procedure. Patients lost to follow up were censored at the last date known to be alive and patients undergoing orthotopic liver transplantation were censored at the date of transplantation.

To assess differences among subgroups (aetiology, indication, bilirubin, ascites, CPS, MELD) in survival time, log rank tests, the Mann-Whitney U test, and Kaplan-Meier analyses were performed. To obtain more precise results, statistical analysis was carried out using the Child-Pugh score and not the Child-Pugh stadium.
Table 1 Demographic, clinical, and biochemical features in patients undergoing elective transjugular intrahepatic portosystemic shunt

\begin{tabular}{|c|c|c|c|c|}
\hline \multirow[b]{2}{*}{ Demographic } & \multirow[t]{2}{*}{$\mathrm{n}$} & \multirow[t]{2}{*}{$\%$} & \multicolumn{2}{|c|}{ Mean (SD) } \\
\hline & & & & \\
\hline Age (y) & 475 & & 56 & $(10.6)$ \\
\hline \multicolumn{5}{|l|}{ Cause of cirrhosis } \\
\hline Alcoholic & 320 & 67.4 & & \\
\hline Virus related & 68 & 14.3 & & \\
\hline Cholestatic & 5 & 1.1 & & \\
\hline Other & 25 & 5.2 & & \\
\hline Missing data & 57 & 12 & & \\
\hline \multicolumn{5}{|l|}{ Clinical } \\
\hline \multicolumn{5}{|l|}{ Ascites (\%) } \\
\hline 1 None & 68 & 14.3 & & \\
\hline 2 Treatable within 1 week & 107 & 22.5 & & \\
\hline 3 Tense ascites & 138 & 29 & & \\
\hline Missing data & 162 & 34.2 & & \\
\hline \multicolumn{5}{|l|}{ Biochemical } \\
\hline Albumin (g/l) & 308 & & 35.8 & (9.2) \\
\hline Serum bilirubin (mg/dl) & 371 & & 2.8 & (3.8) \\
\hline Serum creatinine $(\mathrm{mg} / \mathrm{dll})$ & 377 & & 1.2 & (0.9) \\
\hline INR for prothrombin time & 372 & & 1.5 & $(0.4)$ \\
\hline Prothrombin time (\%) & 372 & & 56.6 & (17.7) \\
\hline \multicolumn{5}{|l|}{ Child-Pugh classification } \\
\hline Score & 238 & & 8.7 & (2) \\
\hline A & 76 & & 6.5 & (0.6) \\
\hline$B$ & 109 & & 8.8 & $(0.8)$ \\
\hline C & 53 & & 11.6 & $(0.7)$ \\
\hline \multicolumn{5}{|l|}{ MELD } \\
\hline Score & 369 & & 7.02 & $(5.83)$ \\
\hline
\end{tabular}

\section{Multivariate analysis for survival}

The main statistical analysis was a Cox proportional hazards regression model. To choose a small set of variables that adequately predicted survival, we used a stepwise Cox model. The significance level of entering or leaving the model was set at $p=0.05$. The candidate variables were the factors shown in table 2 (without CPS, TMS, or MELD because these scores are calculated by combinations of the individual variables listed) that were significant $(p<0.05)$ in a univariate Cox model. To lessen the influence of extreme laboratory values, some quantitative variables were transformed to their natural logarithms. The variables yielded by the Cox model were available in more patients than entered the stepwise model and therefore a further non-stepwise model using only these variables was calculated to reduce the number of missing cases and to obtain more precise results.

\section{Multivariate analysis for scores only}

To compare the scores with each other in multivariate analysis, stepwise models using MELD, TMS, and CPS as independent variables for overall survival (Cox analysis) and for three month survival (logistic regression model) were performed.

\section{Predictive power of MELD/CPS (ROC)}

To compare the accuracy of the two scores as predictors of survival within one year after TIPS, the concordance c statistic (area under the receiver operating characteristic curve (ROC)) was calculated. This statistic may vary from 0 to 1 , with 1 indicating perfect discrimination and 0.5 indicating what is expected by chance alone. For this analysis, death within 1,3 , or 12 months, respectively, was recorded as event. Patients who survived longer than these periods were recorded as nonevents; patients who had a shorter follow up were dropped from this analysis.

The method of DeLong and colleagues ${ }^{28}$ was used to estimate significant differences between MELD and CPS. The concordance c statistic was applied for one month, three month, and one year survival. 
Table 2 Univariate assessment of risk factors for death among elective transjugular intrahepatic portosystemic shunt patients

\begin{tabular}{lllll}
\hline & \multicolumn{3}{l}{ Cox (overall survival) } & \\
\cline { 2 - 5 } Parameter & Patients (n) & $\begin{array}{l}\text { Regression } \\
\text { coefficient }\end{array}$ & $\begin{array}{l}\text { Standard } \\
\text { error }\end{array}$ & $\mathrm{p}$ Value \\
\hline Sex (male) & 471 & -0.15 & 0.15 & 0.32 \\
Age & 475 & 0.019 & 0.006 & 0.002 \\
Log (creatinine) & 377 & 0.499 & 0.152 & 0.001 \\
Log (INR) & 372 & 0.604 & 0.294 & 0.04 \\
Log (bilirubin) & 371 & 0.268 & 0.098 & 0.006 \\
Prothrombin time & 372 & -0.008 & 0.005 & 0.095 \\
Albumin & 308 & -0.034 & 0.01 & 0.001 \\
Cause of cirrhosis & 408 & -0.037 & 0.193 & 0.85 \\
Indication (bleeding) & 420 & 0.346 & 0.162 & 0.033 \\
Aetiology (alcoholic) & 387 & 0.095 & 0.12 & 0.636 \\
Ascites (grade 3) & 313 & 0.362 & 0.116 & 0.002 \\
\hline
\end{tabular}

Cox proportional hazards regression model was performed to assess risk factors for overall survival. Positive coefficients imply that the risk of death increases with increasing values of risk factor. The $p$ values reflect the role of chance in these findings. The relative risk attributable to a risk factor can be estimated from the regression coefficient.

INR, international normalised ratio.

Validation of the original MELD formula

To validate the coefficients of the formula for calculating TMS, a non-stepwise Cox regression model was performed with "log(creatinine)", " $\log ($ bilirubin)", "log(INR)", and "cause of cirrhosis" as independent variables.

\section{Other statistical tests}

For correlation analyses between age, creatinine, INR, prothrombin time, albumin, bilirubin, ascites, TMS, MELD, and CPS, Spearman's correlation coefficients were calculated.

We used the SAS statistical software system (version 8.2; SAS Institute Inc., Cary, North Carolina, USA) to carry out the calculations. A p value $<0.05$ were considered to indicate statistical significance.

\section{RESULTS}

\section{Clinical outcome}

A total of 319 patients underwent a TIPS procedure for prevention of variceal rebleeding and 102 for treatment of refractory ascites. In 54 elective TIPS patients the indication for TIPS placement could not be evaluated. Two hundred and thirty patients died during follow up, 75 within three months of TIPS placement, and 21 underwent liver transplantation. Median follow up was 5.2 years (range 0.003-9.7) and median survival was 4.6 years (range $0-7.8$ years). The three month, one year, three year, and five year survival rates were $84 \%, 73 \%$, $57 \%$, and $48 \%$, respectively (fig 1). Over the years, the age of patients undergoing TIPS implantation (year of implantation $\times a g e$, Spearman's correlation $r=0.006$ ) as well as the severity of disease (year of implantation $\times$ CPS, $r=0.073$ ), reflected by the CPS, did not change.

\section{Survival according to disease aetiology or TIPS indication}

Patients with alcoholic cirrhosis and virus related cirrhosis, the two main disease aetiologies, had similar survival rates $(p=0.636)$ (fig 2A). Patients undergoing TIPS for refractory ascites had significantly poorer survival than patients with variceal rebleeding $(\mathrm{p}=0.032)$ (fig $2 \mathrm{~B})$. They also had more advanced liver disease (Mann-Whitney $\mathrm{U}$ test, $\mathrm{p}<0.0001$ ).

\section{Survival according to CPS and MELD}

CPS divided patients into three classes with significantly different survival rates $(\mathrm{p}=0.001)$. The three month survival rates for CPS classes A, B, and C were $88 \%, 89 \%$, and $62 \%$, respectively (fig $3 \mathrm{~A}$ ). For classes $\mathrm{A}, \mathrm{B}$, and $\mathrm{C}$, three year survival rates were $73 \%, 59 \%$, and $46 \%$, and five year survival rates $64 \%, 57 \%$, and

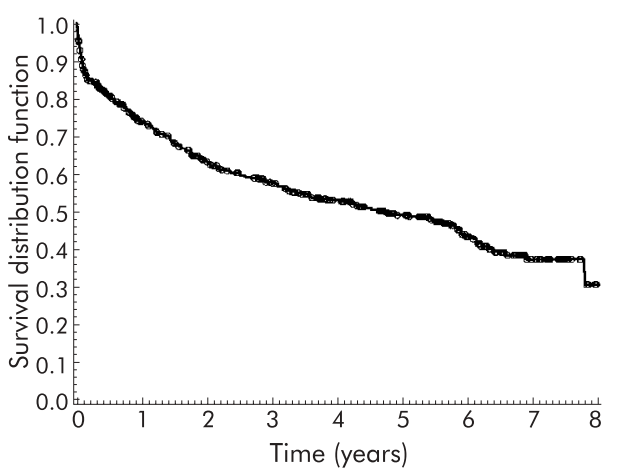

Figure 1 Survival of all patients $(n=475)$ included in the analysis. Symbols indicate patients who were censored on the last date known to be alive or on the date of liver transplantation.

$36 \%$, respectively. Overall survival did not differ between CPS classes A and B whereas survival of patients of class C was poorer $(p=0.009)$ than that of patients of class B. Sixty per cent of patients with MELD $>18(n=17)$, the cut off for high risk patients in the MELD publication, ${ }^{21}$ died within three months of TIPS placement and had a worse life expectancy $(\mathrm{p}=0.002)$ than patients with MELD $\leqslant 18$ (fig 3B). Patients with a MELD score of $>14$ had a median survival of $<90$ days. Patients with a CPS $>11$ also had a median survival $<90$ days.

\section{Survival according to date of TIPS}

Dividing patients into two groups (group $1, \mathrm{n}=229$; group 2 , $\mathrm{n}=246$ ) according to date of TIPS (TIPS implantation before and after 1 January 1996) yielded similar survival rates for both groups $(\mathrm{p}=0.739)$.

\section{Survival according to single determinants}

Univariate analysis for overall survival

Increasing levels of serum creatinine, INR, bilirubin, age, TIPS for refractory ascites, and presence of refractory ascites significantly increased the risk of death. Decreased levels of serum albumin also increased the risk of mortality (table 2).

Multivariate analysis for overall survival (Cox regression) Serum creatinine (step 1), bilirubin (step 2), patient age (step 3), and intractable ascites (step 4) were independent predictors of survival (table 3 ).

These variables were available in more patients than entered the stepwise model, and therefore a further nonstepwise model using only the independent predictors was 

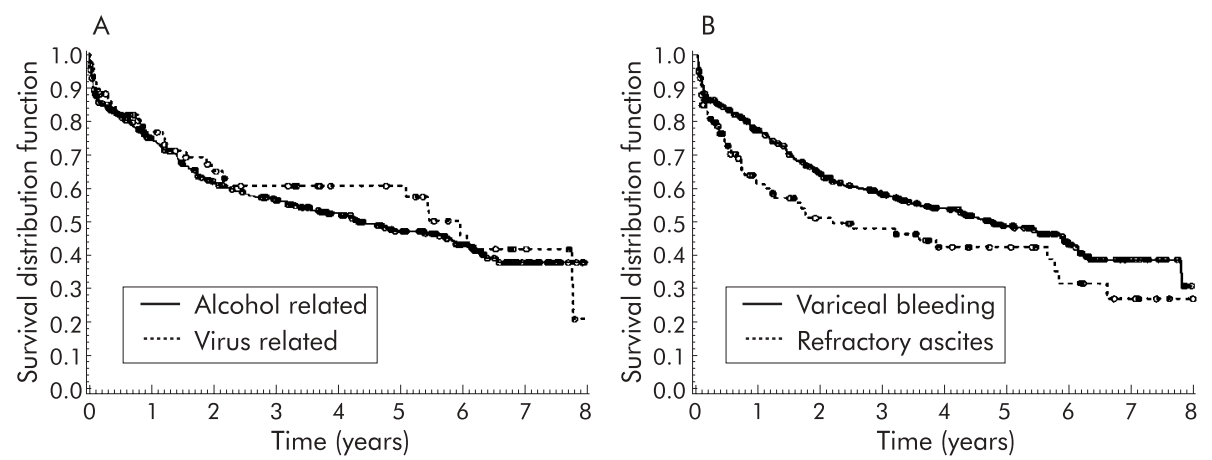

Figure 2 (A) Survival according to underlying liver disease. Patients with alcohol and virus related disease had similar survival rates $(\mathrm{p}=0.636)$. (B) Survival according to indication for transjugular intrahepatic portosystemic shunt (TIPS). Patients undergoing TIPS for prevention of variceal bleeding had a significantly $(p=0.032)$ better survival than patients undergoing TIPS for therapy of refractory ascites.
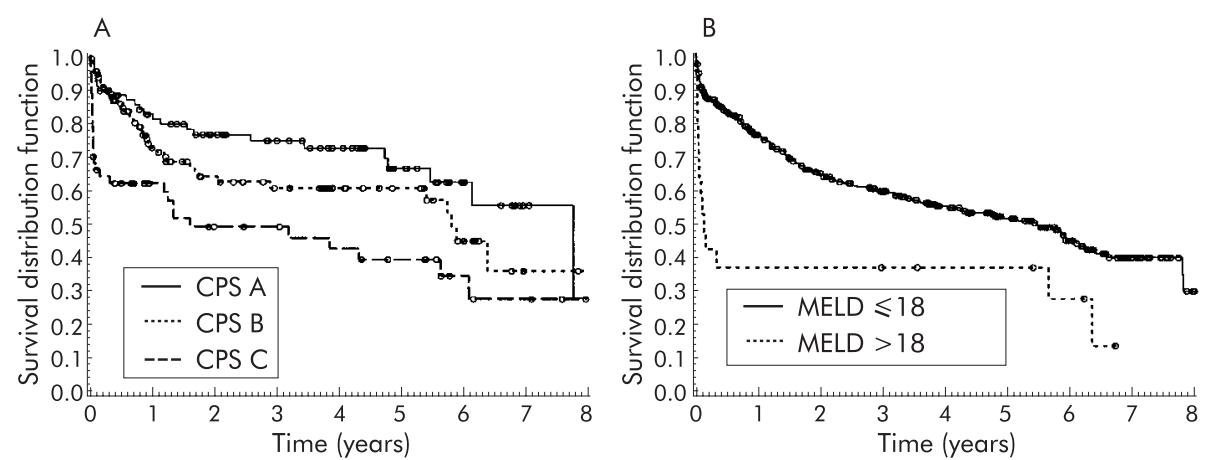

Figure 3 (A) Kaplan-Meier plot according to Child-Pugh score (CPS) class (A $\vee B \vee C)$. CPS divided patients into groups with significantly different survival rates $(p=0.0001)$. (B) Kaplan-Meier plot according to the model for end stage liver disease (MELD) score. Patients with a high risk of death within three months of transjugular intrahepatic portosystemic shunt placement (MELD >18) had highly significant $(p=0.002)$ poorer survival rates than low risk patients (MELD $\leqslant 18)$.

Table 3 Overall survival model for patients undergoing elective transjugular intrahepatic portosystemic shunt (TIPS). Stepwise Cox model to assess independent predictors of death after TIPS implantation $(n=234$, death=94) and validation of the variables yielded by this stepwise Cox model in a non-stepwise analysis to reduce the number of missing cases $(n=304$, death $=133)$

\begin{tabular}{|c|c|c|c|c|c|c|c|c|c|}
\hline \multirow[b]{2}{*}{ Variable } & \multicolumn{5}{|c|}{ Stepwise Cox model $(n=234)$} & \multicolumn{4}{|c|}{ Validation $(n=304)$} \\
\hline & Step & Hazard ratio & $95 \% \mathrm{Cl}$ & & p Value & Hazard ratio & $95 \% \mathrm{Cl}$ & & $\mathrm{p}$ Value \\
\hline Creatinine & 1 & 2.238 & 1.564 & 3.464 & $<0.001$ & 1.728 & 1.217 & 2.453 & 0.002 \\
\hline Bilirubin & 2 & 1.538 & 1.199 & 1.973 & 0.001 & 1.351 & 1.084 & 1.684 & 0.007 \\
\hline Age & 3 & 1.023 & 1.004 & 1.042 & 0.016 & 1.025 & 1.008 & 1.042 & 0.003 \\
\hline Refractory ascites & 4 & 1.608 & 1.096 & 2.576 & 0.017 & 1.514 & 1.067 & 2.147 & 0.02 \\
\hline
\end{tabular}

calculated to reduce the number of missing cases. This yielded more precise results reflected by shorter confidence limits. In this analysis, the parameter estimate of creatinine was found to be much lower than in the stepwise model $(0.84 v 0.55)$, and the regression coefficients for the other parameters changed slightly (table 3$)$.

Multivariate analysis for three month survival

For prediction of three month survival, no parameters other than those in the Cox regression were identified, and the weight of the variables changed only slightly compared with overall survival.

\section{CPS versus MELD score}

Multivariate analysis of CPS, TMS, and MELD as three month and overall survival predictors yielded MELD as a single independent predictor for overall (Cox analysis: parameter estimate 0.053 , standard error $0.012, \mathrm{p}<0.0001$ ) as well as for three month survival (log rank test: parameter estimate 0.19, standard error $0.036, \mathrm{p}<0.0001)$.

\section{Model validation of CPS and MELD (c statistics)}

The accuracy of the two scores for one month, three months, and one year survival was compared. The discrimination powers of MELD (c statistics: 0.73, 0.72, 0.66 for one month, three month, and one year survival) were not significantly different from the discrimination powers of CPS at the same time points (c statistics: $0.78,0.70,0.66$ ). The area under the curves showed a decrease in the predictive value for both scores during follow up. The ROC for three month survival is shown in fig 4 .

Using a cut off of a predicted probability of death of 0.25 within three months, the sensitivity of MELD was $40 \%$, specificity $90 \%$, positive predictive value $46 \%$, and negative predictive value $87 \%$. Table 4 shows the use of the MELD model in our patients; with a MELD predicted mortality of 


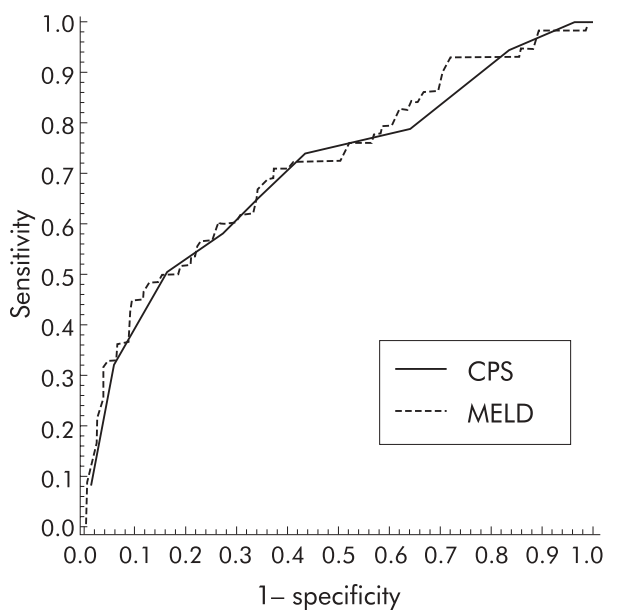

Figure 4 Receiver operating characteristic curve for Child-Pugh score (CPS) and model for end stage liver disease (MELD) for three month survival. The area under the curve (AUC) was 0.7 for CPS and 0.72 for MELD, which was not significantly different.

Table 4 Utility of the MELD formula in our patients in predicting three month mortality post transjugular intrahepatic portosystemic shunt

\begin{tabular}{lcccl}
\hline & \multicolumn{4}{c}{ Predicted mortality by MELD formula } \\
\cline { 2 - 5 } & $\mathbf{0 - 2 5 \%}$ & $\mathbf{2 5 - 5 0 \%}$ & $50-75 \%$ & $\mathbf{7 5 - 1 0 0 \%}$ \\
\hline No of patients & 221 & 106 & 18 & 5 \\
No of deaths & 21 & 23 & 10 & 3 \\
Observed mortality & $9.5 \%$ & $21.7 \%$ & $55.56 \%$ & $60 \%$ \\
\hline
\end{tabular}

$0-25 \%, 9.5 \%$ of patients died, whereas in the group with a predicted mortality of $75-100 \%, 60 \%$ died.

Both a threshold of 14 for MELD and 11 for CPS had a specificity of $94 \%$ but a sensitivity of 34\% for MELD and 33\% for CPS.

Validation of the formula of Malinchoc and colleagues ${ }^{20}$ We validated the original MELD formula by recalculating it using our patient data. Of the four variables included in the analysis (serum creatinine, bilirubin, INR, cause of cirrhosis), only creatinine and bilirubin correlated significantly with survival. The regression coefficient entered into our formula was lower for creatinine $(0.55 v 0.96)$ and nearly equal for bilirubin ( $0.31 v 0.39)$ compared with Malinchoc's coefficients (table 5).

\section{DISCUSSION}

Establishment of prognostic factors is the key towards evaluating clinical interventions in any disease. The most commonly used prognostic model in patients with cirrhosis is the CPS. Modifications of CPS either by adding new variables or by employing more sophisticated measures did not improve its precision ${ }^{29-34}$ or applicability. ${ }^{35-38} \mathrm{~A}$ new approach is the MELD formula which is used mostly in patients with end stage cirrhosis awaiting liver transplantation. In our study, the predictive value of baseline patient characteristics and determinants of both scores were evaluated in a large cohort of 475 patients who underwent elective TIPS placement within 10 years. In previous publications, all individual variables of both prognostic scores and the scores themselves have been found to correlate with survival. ${ }^{4} 7^{15-19} 21$ In our study, all individual determinants of CPS and MELD (except for the cause of cirrhosis) correlated with survival by univariate analysis, and multivariate Cox analysis identified four independent prognostic factors for overall survival: level of serum creatinine was the strongest predictor, followed by bilirubin, patient age, and presence of refractory ascites. The key finding was identification of renal function as the best predictor of survival in patients with end stage liver cirrhosis.

End stage liver disease or events such as bleeding, infection, and high dose diuretic therapy have effects on renal function and may cause functional renal impairment. In patients presenting with a high CPS class, the impact of impaired renal function on survival is well known. ${ }^{33}{ }^{39}$ Seventy seven per cent of our patients were of Child-Pugh class A or B, and multivariate analysis showed that levels of serum creatinine correlated with survival in patients with apparently normal renal function (serum creatinine within the normal range). This finding suggests that the level of creatinine is a prognostic marker even in patients with good liver synthetic function and normal renal function. TIPS implantation leads to improvement in renal function ${ }^{40}$ and to reduction or resolution of ascites. ${ }^{2}$ Despite the therapeutic benefit of TIPS, creatinine levels were an independent predictor of death after TIPS implantation.

INR correlated with survival in univariate analysis but multivariate analysis showed that the prognostic impact of coagulation parameters on overall survival was not an independent predictor in our patients. INR was used in the MELD score because it can be used as an uniform coagulation parameter all over the world. ${ }^{41}{ }^{42}$ Although coagulation parameters are sensible individual markers of liver synthetic function, the value of INR - also supported by our study - as a parameter of liver function is, at the least, controversial. ${ }^{23} 4344$

Multivariate analysis yielded two variables of MELD (creatinine and bilirubin) as well as two variables of CPS (ascites and bilirubin) as predictors of survival. We therefore compared both scores with each other. MELD was found to be an independent predictor of overall survival because of the strong impact of creatinine on long term survival, which is not a variable of the CPS. We also tried to improve the CPS by adding serum creatinine as a sixth variable but were unable to improve it significantly beyond MELD. ${ }^{45}$ With longer follow up, a decrease in the predictive power of both scores was observed. Unpredictable complications such as stenoses of TIPS, development of malignant tumours, or non-liver related events influence the long term predictive value of both scores. The aim of the original MELD study ${ }^{20}$ and the MELD study ${ }^{21}$

Table 5 Validation of the weight of the variables used in the original MELD formula

\begin{tabular}{llllccc}
\hline Variable & $\begin{array}{l}\text { Regression } \\
\text { coefficient } \\
\text { (MELD) }\end{array}$ & $\begin{array}{l}\text { Standard } \\
\text { error } \\
\text { (MELD) }\end{array}$ & $\begin{array}{l}\text { Regression } \\
\text { coefficient } \\
\text { (Vienna) }\end{array}$ & $\begin{array}{l}\text { Standard } \\
\text { error } \\
\text { (Vienna) }\end{array}$ & $\begin{array}{l}\text { 95\% Regression coefficient } \\
\text { confidence limits (Vienna) }\end{array}$ \\
\hline Creatinine & 0.957 & 0.142 & 0.536 & 0.156 & 0.230 & 0.842 \\
Bilirubin & 0.378 & 0.117 & 0.308 & 0.107 & 0.098 & 0.516 \\
INR & 1.12 & 0.331 & 0.293 & 0.334 & -0.362 & 0.948 \\
Cause of cirrhosis & 0.643 & 0.211 & 0.067 & 0.208 & -0.341 & 0.474 \\
\hline
\end{tabular}

The table shows the comparison of the regression coefficients and the standard errors between the MELD model and the Vienna data. The standard errors were nearly equal which means that both studies included comparable patients. 
was not to predict overall survival, but three month survival, ${ }^{20}$ and one month, three month, and one year survival, ${ }^{21}$ respectively. For these time points, no significant difference in the predictive power between the two scores was found in our patients. In the MELD study, ${ }^{21}$ the area under the curve for three month survival for 282 hospitalised cirrhotic patients was found to be 0.84 (CPS) and 0.87 (MELD), which indicates excellent diagnostic accuracy ${ }^{21}$ for both scores. In these patients, as well as in our patients, both scores were shown to be equally predictive.

Although both scores were able to predict survival in our patients, both have several shortcomings. The drawbacks of CPS are well known ${ }^{36}$ and led to the development of the MELD score. This study demonstrates that MELD also has several shortcomings. The original MELD formula ${ }^{20}$ was modified by dropping the variable "cause of cirrhosis". ${ }^{21}{ }^{4}{ }^{47}$ However, the formula was not reassessed nor was the weight for the regression coefficients re-evaluated. Therefore, we recalculated the MELD formula and evaluated regression coefficients. Although creatinine was the strongest predictor of survival in our patients, its weight seems to have been overestimated in the original formula. This may be caused by a smaller number of patients in the sample of the MELD publication ${ }^{20}$ compared with our patient sample. While the value of bilirubin was confirmed, neither INR nor the cause of cirrhosis had any prognostic significance in our patients.

Previously, a MELD score of 18 was identified as a threshold for high risk patients with a median survival time of three months or less, but only $19 \%$ of patients who died within three months after TIPS placement had a MELD score $>18$. This clearly shows a poor ability of MELD to rank patients into groups of high and low risk. In our patients, the threshold for MELD was found to be lower $(>14)$ than the original threshold (>18), increasing the sensitivity of MELD from 19\% to $34 \%$. For CPS, we identified a threshold of $>11$ for high risk patients with a median survival of three months or less. Using this cut off, $33 \%$ of patients who died within three months after TIPS had a CPS > 11 (sensitivity 33\%, specificity 94\%). Our data therefore suggest that these cut off levels may be used to help with the decision of who should receive TIPS.

In patients undergoing TIPS, a prognostic score should stratify patients into those with a good prognosis and those who probably should not undergo the procedure because of the high risk of early death. The advantage of MELD is its reproducibility, objectivity, and ability to stratify patients according to their individual risk. Thus MELD appears to be better than CPS as a disease severity index for allocating organs for patients undergoing liver transplantation. As TIPS is available for all patients and there is no need to rank these patients according to disease severity, CPS seems to be a more than adequate score for patients undergoing TIPS. CPS is well established, easy to perform at the bedside, and can reliably stratify patients undergoing TIPS into high and low risk. While the CPS is seasoned, it is not yet ready to retire.

\section{Authors' affiliations}

B Angermayr, J Pidlich, M Wichlas, A Kreil, M Schmid, A Ferlitsch, E Lipinski, P Ferenci, A Gangl, M Peck-Radosavljevic, Department of Gastroenterology and Hepatology, University of Vienna Medical School, Vienna, Austria

M Ceina, J Lammer, Department of Interventional Radiology, University of Vienna Medical School, Vienna, Austria

F Karnel, Department of Radiology, Kaiser Franz-Josef Spital, Vienna,

Austria

M Gschwantler, Department of Internal Medicine, Krankenhaus

Rudolfstiftung, Vienna, Austria

F Koenig, Department of Medical Statistics, University of Vienna Medical

School, Vienna, Austria

H Mendel, Department of Radiology, Krankenhaus Lainz, Vienna,

Austria

H Brunner, Department of Internal Medicine, Krankenhaus Lainz, Vienna, Austria

L Pichler, Department of Radiology, Donauspital, Vienna, Austria

\section{REFERENCES}

1 Rossle M, Haag K, Ochs A, et al. The transjugular intrahepatic portosystemic stent- shunt procedure for variceal bleeding. N Engl J Med 1994:330:165-71.

2 Rossle M, Ochs A, Gulberg V, et al. A comparison of paracentesis and transjugular intrahepatic portosystemic shunting in patients with ascites. NEngl J Med 2000;342:1701-7.

3 Sanyal AJ. The use and misuse of transjugular intrahepatic portasystemic shunts. Curr Gastroenterol Rep 2000;2:61-71

4 Ochs A, Rössle M, Haag K, et al. The transjugular intrahepatic portosystemic stent- shunt procedure for refractory ascites (published erratum appears in N Engl J Med 1995;332:1587). N Engl J Med 1995;332:1192-7

5 Rössle M, Deibert $P$, Haag K, et al. Randomised trial of transjugular-intrahepatic- portosystemic shunt versus endoscopy plus propranolol for prevention of variceal rebleeding. Lancet 1997; 349: 1043-9.

6 Peck-Radosavlievic M, Pidlich J. Der transjuguläre intrahepatische portosystemische Shunt (TIPS). Wien Klin Wochenschr 2000;1 12:947-54.

7 Gines P, Quintero E, Arroyo V, et al. Compensated cirrhosis: natural history and prognostic factors. Hepatology 1987;7:122-8.

8 Helton WS, Belshaw A, Althaus S, et al. Critical appraisal of the angiographic portacaval shunt (TIPS). Am J Surg 1993;165:566-71 9 Jalan R, Elton RA, Redhead DN, et al. Analysis of prognostic variables in the prediction of mortality, shunt failure, variceal rebleeding and encephalopathy following the transjugular intrahepatic portosystemic stent-shunt for variceal haemorrhage. J Hepatol 1995:23:123-8.

10 LaBerge JM, Somberg KA, Lake JR, et al. Two-year outcome following transjugular intrahepatic portosystemic shunt for variceal bleeding: results in 90 patients. Gastroenterology 1995;108:1143-51.

11 Stanley AJ, Jalan R, Forrest EH, et al. Longterm follow up of transjugular intrahepatic portosystemic stent shunt (TIPSS) for the treatment of portal hypertension: results in 130 patients. Gut 1996;39:479-85.

12 Sahagun G, Benner KG, Saxon R, et al. Outcome of 100 patients after transjugular intrahepatic portosystemic shunt for variceal hemorrhage. Am J Gastroenterol 1997;92:1444-52.

13 Rossle M, Siegerstetter V, Huber M, et al. The first decade of the transjugular intrahepatic portosystemic shunt (TIPS): state of the art. Liver 1998:18:73-89.

14 Pugh RN, Murray-Lyon IM, Dawson JL, et al. Transection of the oesophagus for bleeding oesophageal varices. Br J Surg 1973:60:646-9.

15 Rajan DK, Haskal ZJ, Clark TW. Serum bilirubin and early mortality after transjugular intrahepatic portosystemic shunts: results of a multivariate analysis. J Vasc Interv Radiol 2002;13:155-61.

16 Cooper GS, Bellamy P, Dawson NV, et al. A prognostic model for patients with end-stage liver disease. Gastroenterology 1997:113:1278-88.

17 Poynard T, Zourabichvili O, Hilpert G, et al. Prognostic value of total serum bilirubin/gamma-glutamyl transpeptidase ratio in cirrhotic patients. Hepatology 1984;4:324-7

18 Christensen E, Schlichting P, Faverholdt L, et al. Prognostic value of Child-Turcotte criteria in medically treated cirrhosis. Hepatology $1984 ; 4: 430-5$

19 Infante-Rivard C, Esnaola S, Villeneuve JP. Clinical and statistical validity of conventional prognostic factors in predicting short-term survival among cirrhotics. Hepatology 1987;7:660-4

20 Malinchoc M, Kamath PS, Gordon FD, et al. A model to predict poor survival in patients undergoing transjugular intrahepatic portosystemic shunts. Hepatology 2000;31:864-71.

21 Kamath PS, Wiesner RH, Malinchoc $M$, et al. A model to predict survival in patients with end-stage liver disease. Hepatology 2001;33:464-70

22 Arroyo V, Gines P, Gerbes AL, et al. Definition and diagnostic criteria of refractory ascites and hepatorenal syndrome in cirrhosis. International Ascites Club. Hepatology 1996;23:164-76.

23 van den Besselaar AM. Precision and accuracy of the international normalized ratio in oral anticoagulant control. Haemostasis 1996;26(suppl 4):248-65

24 Angermayr B, Cejna M, Schoder $M$, et al. Transjugular intrahepatic portosystemic shunt for treatment of portal hypertension due to extramedullary hematopoiesis in idiopathic myelofibrosis. Blood 2002;99:4246-7.

25 Cejna M, Peck-Radosavljevic M, Schoder M, et al. Repeat interventions for maintenance of transiugular intrahepatic portosystemic shunt function in patients with Budd-Chiari syndrome. J Vasc Interv Radiol 2002;13:193-9.

26 LaBerge JM, Ring EJ, Gordon RL, et al. Creation of transjugular intrahepatic portosystemic shunts with the wallstent endoprosthesis: results in 100 patients. Radiology 1993;187:413-20.

27 Ceina M, Thurnher S, Pidlich J, et al. Primary implantation of polyester-covered stent-grafts for transjugular intrahepatic portosystemic stent shunts (TIPSS): A pilot study. Cardiovasc Intervent Radiol 1999;22:305-10.

28 DeLong ER, DeLong DM, Clarke-Pearson DL. Comparing the areas under two or more correlated receiver operating characteristic curves: a nonparametric approach. Biometrics 1988;44:837-45.

29 Merkel C, Bolognesi M, Bellon S, et al. Aminopyrine breath test in the prognostic evaluation of patients with cirrhosis. Gut 1992;33:836-42

30 Beuers U, Jager F, Wahllander A, et al. Prognostic value of the intravenous 14C-aminopyrine breath test compared to the Child-Pugh 
score and serum bile acids in 84 cirrhotic patients. Digestion 1991;50:212-18.

31 Arrigoni A, Gindro T, Aimo G, et al. Monoethylglicinexylidide test: a prognostic indicator of survival in cirrhosis. Hepatology 1994;20:383-7.

32 Salerno F, Borroni G, Moser P, et al. Prognostic value of the galactose test in predicting survival of patients with cirrhosis evaluated for liver transplantation. A prospective multicenter Italian study. AISF Group for the Study of Liver Transplantation. Associazione Italiana per lo Studio del Fegato. J Hepatol 1996;25:474-80

33 Abad-Lacruz A, Cabre E, Gonzalez-Huix F, et al. Routine tests of renal function, alcoholism, and nutrition improve the prognostic accuracy of Child-Pugh score in nonbleeding advanced cirrhotics. Am J Gastroenterol 1993;88:382-7.

34 Adler $M$, Verset $D$, Bouhdid $H$, et al. Prognostic evaluation of patients with parenchymal cirrhosis. Proposal of a new simple score. J Hepatol 1997;26:642-9.

35 Reuben A. Child comes of age. Hepatology 2002;35:244-5

36 Conn HO. A peek at the Child-Turcotte classification. Hepatology 1981;1:673-6.

37 Albers I, Hartmann H, Bircher J, et al. Superiority of the Child-Pugh classification to quantitative liver function tests for assessing prognosis of liver cirrhosis. Scand J Gastroenterol 1989:24:269-76.

38 Forman LM, Lucey MR. Predicting the prognosis of chronic liver disease: an evolution from child to MELD. Mayo End-stage Liver Disease. Hepatology 2001;33:473-5.
39 Arroyo V, Bosch J, Gaya-Beltran J, et al. Plasma renin activity and urinary sodium excretion as prognostic indicators in nonazotemic cirrhosis with ascites. Ann Intern Med 1981;94:198-201.

40 Wong F, Sniderman K, Liu P, et al. The mechanism of the initial natriuresis after transjugular intrahepatic portosystemic shunt. Gastroenterology 1997;1 12:899-907.

41 Deitcher SR. Interpretation of the international normalised ratio in patients with liver disease. Lancet 2002;359:47-8.

42 Kovacs MJ, Wong A, MacKinnon K, et al. Assessment of the validity of the INR system for patients with liver impairment. Thromb Haemost 1994; $71: 727-30$

43 Denson KW, Reed SV, Haddon ME. Validity of the INR system for patients with liver impairment. Thromb Haemost 1995;73:162.

44 Robert A, Chazouilleres $O$. Prothrombin time in liver failure: time, ratio, activity percentage, or international normalized ratio? Hepatology 1996;24:1392-4.

45 Angermayr B, Koenig F, Cejna $M$, et al. Creatinine-modified Child-Pugh-Score (CPSC) compared with MELD-Score to predict survival in patients undergoing TIPS (abstract). Hepatology 2002;36:860.

46 Freeman RB Jr, Wiesner RH, Harper A, et al. The new liver allocation system: Moving toward evidence-based transplantation policy. Liver Transp/ 2002;8:851-8.

47 Wiesner RH, McDiarmid SV, Kamath PS, et al. MELD and PELD: application of survival models to liver allocation. Liver Transpl $2001 ; 7: 567-80$ 